\title{
UNIVERSAL MAPPING PROPERTIES OF SOME PSEUDOVALUATION DOMAINS AND RELATED QUASILOCAL DOMAINS
}

\author{
AHMED AYACHE, DAVID E. DOBBS, AND OTHMAN ECHI
}

Received 24 January 2005; Revised 5 January 2006; Accepted 22 January 2006

If $(R, M)$ and $(S, N)$ are quasilocal (commutative integral) domains and $f: R \rightarrow S$ is a (unital) ring homomorphism, then $f$ is said to be a strong local homomorphism (resp., radical local homomorphism) if $f(M)=N$ (resp., $f(M) \subseteq N$ and for each $x \in N$, there exists a positive integer $t$ such that $\left.x^{t} \in f(M)\right)$. It is known that if $f: R \rightarrow S$ is a strong local homomorphism where $R$ is a pseudovaluation domain that is not a field and $S$ is a valuation domain that is not a field, then $f$ factors via a unique strong local homomorphism through the inclusion map $i_{R}$ from $R$ to its canonically associated valuation overring $(M: M)$. Analogues of this result are obtained which delete the conditions that $R$ and $S$ are not fields, thus obtaining new characterizations of when $i_{R}$ is integral or radicial. Further analogues are obtained in which the "pseudovaluation domain that is not a field" condition is replaced by the APVDs of Badawi-Houston and the "strong local homomorphism" conditions are replaced by "radical local homomorphism."

Copyright @ 2006 Hindawi Publishing Corporation. All rights reserved.

\section{Introduction}

Pseudovaluation domains (for short, PVDs) were introduced by Hedstrom and Houston [21]. PVDs and their generalizations to the context of rings with nontrivial zero-divisors have been studied extensively (see, e.g., [1, 2, 4-11, 14-17, 19, 21-23, 25, 26]), but none of these studies featured categorical aspects. Recently, in [3], we presented a categorical study, emphasizing reflective subcategories, of some classes of quasilocal (commutative integral) domains that are "close" to PVDs. These included the domains that we called $\mathscr{T}$-domains, and pseudo- $\mathscr{T}$-domains (whose definitions are recalled later in this section). In [3], we obtained two results showing that certain naturally occurring inclusions of categories admit left adjoint functors. For convenience, equivalent formulations of those results are stated in Theorems 2.1 and 2.2 in terms of universal mapping properties. The morphisms that figure in these statements are the special type of (unital) local ring homomorphism $f:(R, M) \rightarrow(S, N)$ such that $f(M)=N$; we called such an $f$ a "strong local homomorphism" (or an sl-homomorphism) in [3]. Unfortunately, the techniques in [3] 
require that the domains considered in Theorems 2.1 and 2.2 not be fields. The main goal of the present paper is to investigate the effect on results like these theorems if one allows the relevant PVDs, valuation domains, $\mathscr{T}$-domains , and pseudo- $\mathscr{T}$-domains to be fields.

Example 2.3 shows that additional hypotheses are needed if one is to obtain positive results while dealing with fields. Our main positive results are given in Theorems 2.5 and 2.8. Loosely stated, these theorems can be summarized by saying that under certain conditions, universal mapping properties characterize when the inclusion map $i_{R}:(R, M) \rightarrow(M: M)$ is radicial (in the sense of [20]), while (not necessarily universal) mapping properties characterize when $i_{R}$ is integral. Although Theorem 2.5 continues the study from [3] of ring homomorphisms $R \rightarrow S$ whose domains $R$ are PVDs, the codomains $S$ of those homomorphisms are generalized from being valuation domains (as they were in Theorem 2.1) to being $\mathscr{T}$-domains. In addition, by replacing the sl-homomorphisms with a more general type of local map that we call a "radical local homomorphism," we obtain in Theorem 2.8 an analogue of Theorem 2.5 in which the PVDs are replaced by the more general almost pseudovaluation domains (or APVDs) that were recently introduced by Badawi and Houston [11].

We close this introduction by giving two paragraphs of background. Following [3], we adopt the following notation and terminology, given a quasilocal domain $(R, M)$ with quotient field $K$. Let $\mathscr{T}(R):=(M: M)=\{x \in K \mid x M \subseteq M\}$, and let $i_{R}: R \hookrightarrow \mathscr{T}(R)$ be the inclusion map. Of course, $\mathscr{T}(R)$ is an overring of $R$, that is, a ring contained between $R$ and $K$. Recall from [2, Proposition 2.5] that $R$ is a PVD if and only if $\mathscr{T}(R)$ is a valuation domain with maximal ideal $M$; furthermore, in this case, we have $\operatorname{Spec}(R)=$ $\operatorname{Spec}(\mathscr{T}(R))$ (where, as usual, $\operatorname{Spec}(A)$ denotes the set of all prime ideals of a commutative ring $A$ ). In particular, if $R$ is a PVD, then $i_{R}$ is an sl-homomorphism. We say that (the ambient quasilocal domain) $R$ is a $\mathscr{T}$-domain if $\mathscr{T}(R)=R$; and a pseudo- $\mathscr{T}$-domain if $\operatorname{Spec}(\mathscr{T}(R))=\operatorname{Spec}(R)$ (as sets). As recorded in [3, Remarks 3.1], it is clear that every valuation domain is a $\mathscr{T}$-domain, every PVD is a pseudo- $\mathscr{T}$-domain, and every $\mathscr{T}$-domain is a pseudo- $\mathscr{T}$-domain. However, [3, Examples 3.2 and 3.3] show that a $\mathscr{T}$-domain need not be a valuation domain and a pseudo- $\mathcal{T}$-domain need not be a PVD.

Finally, to complete the summary of background, we recall two results for motivational purposes. It was observed in [3, Corollary 3.7] that (the ambient quasilocal domain) $R$ is a pseudo- $\mathscr{T}$-domain if and only if there exists a one-to-one sl-homomorphism $u$ from $R$ to a $\mathscr{T}$-domain T. Moreover (by [3, Proposition 3.9]), if $u: R \rightarrow T$ is a one-to-one slhomomorphism from a pseudo- $\mathscr{T}$-domain $R$ to a $\mathscr{T}$-domain $T$ such that neither $R$ nor $T$ is a field, then the following universal mapping property holds: for each $\mathscr{T}$-domain $S$ which is not a field and each sl-homomorphism $f: R \rightarrow S$, there exists a unique slhomomorphism $\tilde{f}: T \rightarrow S$ such that $\tilde{f} u=f$.

\section{Results}

We begin by stating a result from [3] that is the culmination of an "orthogonality" study of pseudovaluation domains. Let PVD denote the category whose objects are the pseudovaluation domains that are not fields with sl-homomorphisms as morphisms, and let VD be the full subcategory of PVD whose objects are all the valuation domains that are not fields. It was shown in [3, Corollary 2.9] that VD is a reflective subcategory of PVD; 
that is, that the inclusion functor VD $\rightarrow$ PVD has a left adjoint functor (and that the reflection functor PVD $\rightarrow$ VD assigns to a pseudovaluation domain $(R, M)$ that is not a field its canonically associated valuation domain $\mathscr{T}(R)$ ). Theorem 2.1 gives an equivalent formulation of [3, Corollary 2.9] in terms of a universal mapping property. Note that this formulation is in the same spirit as the final result [3, Proposition 3.9] that was recalled in the introduction.

Theorem 2.1 [3, Corollary 2.9]. Let $i_{R}: R \hookrightarrow \mathscr{T}(R)$ be the inclusion map from a pseudovaluation domain $R$ to its canonically associated valuation domain $\mathscr{T}(R)$. Suppose also that $R$ is not a field (in which case $\mathcal{T}(R)$ is also not a field). Then the following universal mapping property holds: for each valuation domain $S$ which is not a field and each sl-homomorphism $f: R \rightarrow S$, there exists a unique sl-homomorphism $\tilde{f}: T \rightarrow S$ such that $\tilde{f} i_{R}=f$.

By pursuing the categorical (especially, "orthogonal") work that had led to [3, Corollary 2.9], we obtained in [3, Corollary 3.5] the following analogue: the full subcategory consisting of $\mathscr{T}$-domains that are not fields is reflective in the category of pseudo- $\mathscr{T}$ domains that are not fields (with morphisms, the strong local homomorphisms). In the spirit of Theorem 2.1, we next state an equivalent formulation of [3, Corollary 3.5] in terms of a universal mapping property.

Theorem 2.2 [3, Corollary 3.5]. Let $R$ be a pseudo- $\mathcal{T}$-domain and $i_{R}: R \hookrightarrow \mathscr{T}(R)$ the canonical inclusion map. Suppose also that $R$ is not a field (in which case $\mathscr{T}(R)$ is also not a field). Then the following universal mapping property holds: for each $\mathscr{T}$-domain $S$ which is not a field and each sl-homomorphism $f: R \rightarrow S$, there exists a unique sl-homomorphism $\tilde{f}: T \rightarrow S$ such that $\tilde{f} i_{R}=f$.

Notice that Theorem 2.2 can be considered an analogue of Theorem 2.1 in that we changed the types of rings being considered, by replacing pseudovaluation domains and valuation domains with $\mathscr{T}$-domains and pseudo- $\mathscr{T}$-domains, respectively. The purpose of this paper is to develop additional analogues, this time by enlarging the categories in which the (possibly universal) mapping properties reside. We begin by showing that if one weakens "sl-homomorphism" to "local homomorphism" and permits the codomains of morphisms to be pseudovaluation domains (that are not fields) rather than valuation domains (or, at least, $\mathscr{T}$-domains), then a (universal) mapping property of the kind established in Theorems 2.1 and 2.2 is no longer available.

Example 2.3. There exist a PVD, $(R, M)$, with associated valuation domain $V:=\mathscr{T}(R):=$ $(M: M)$ and inclusion map $i_{R}: R \rightarrow V$, and a local homomorphism $f$ from $R$ to some PVD, $(H, P)$, such that there does not exist a ring homomorphism $g: V \rightarrow H$ such that $g i_{R}=f$.

Let $X, Y$ be analytically independent indeterminates over $\mathbb{C}$. Consider the valuation domain $V:=\mathbb{C}[[X]]=\mathbb{C}+M$, with maximal ideal $M=X V$. Then $R:=\mathbb{R}+M$ is a PVD with canonically associated valuation domain $V$. Put $H:=\mathbb{R}+X \mathbb{C}(Y)[[X]]$. Observe that $H$ is a PVD with associated valuation domain $\mathbb{C}(Y)[[X]]$ and maximal ideal $P:=$ $X \mathbb{C}(Y)[[X]]$. Take $f$ to be the inclusion map $R \hookrightarrow H$. Observe that $f$ is a local homomorphism (but not an sl-homomorphism) since $M \subset P$. It remains only to show that there 
does not exist a ring homomorphism $g: V \rightarrow H$ such that $g i_{R}=f$. In fact, there does not exist a (unital) ring homomorphism $g: V \rightarrow H$, for any such $g$ would have to satisfy $g(i)^{2}=-1$ and a simple order argument confirms that $H$ does not contain any element $h$ such that $h^{2}=-1$.

Thanks to a referee, we can also provide the following second example. This example has the virtue of featuring a field in the role of $H$, and thus explains the need for additional hypotheses (such as $H / P$ being algebraically closed) in Theorems 2.5 and 2.8. Let $R$ and $V$ be as above, but in this new example, take $H:=\mathbb{R}$, identified with $R / M$, and take $f: R \rightarrow H$ to be the canonical surjection. Then one sees as above that there is no ring homomorphism $g: V \rightarrow H$, to complete the proof.

In view of the preceding example, we focus again on sl-(rather than on local) homomorphisms. By doing so, we show in Theorem 2.5(b) that some PVDs have a universal mapping property relative to morphisms with rather general codomains. To prepare for that result, we first develop some field-theoretic material.

As in [24], if $K$ and $L$ are fields and $\sigma: K \rightarrow L$ is a (unital) ring homomorphism (i.e., a unital $K$-algebra homomorphism), we call $\sigma$ an embedding (of $K$ in $L$ ). Embeddings are necessarily one-to-one. Certain field-theoretic embeddings, such as inclusion maps into splitting fields or in particular algebraic closures, have important mapping properties (cf. [24, Theorem 2.8, page 233]). The next lemma collects two such properties which will be used in proving the two (new) theorems in this section. Although Lemma 2.4 may be known, we include a proof for the sake of completeness.

Lemma 2.4. Let $\sigma: K \rightarrow L$ be an embedding of a field $K$ in a field L. (e.g., $K$ could be a subfield of a field $L$, with $\sigma$ the inclusion map from $K$ to $L$.) Then

(a) the following two conditions are equivalent:

(1) for each embedding $\tau: K \rightarrow F$ of $K$ in an algebraically closed field $F$, there exists at least one embedding $\rho: L \rightarrow F$ such that $\rho \sigma=\tau$;

(2) $L$ is an algebraic field extension of $K$;

(b) the following two conditions are equivalent:

(i) for each embedding $\tau: K \rightarrow F$ of $K$ in an algebraically closed field $F$, there exists a unique embedding $\rho: L \rightarrow F$ such that $\rho \sigma=\tau$;

(ii) $L$ is a purely inseparable (algebraic) field extension of $K$.

Proof. For simplicity of notation, we view $\sigma$ and $\tau$ as inclusion maps.

(a) For $(2) \Rightarrow(1)$, apply the above-mentioned mapping property [24, Theorem 2.8, page 233]. If the converse fails, choose an element $Y \in L$ that is transcendental over $K$. Take $F$ to be an algebraic closure of $K$, with $\tau: K \rightarrow F$ as the inclusion map. By (1), there exists an embedding $\rho: L \rightarrow F$ such that $\rho \sigma=\tau$. In particular, $\rho(Y)$ is algebraic over $K$. Hence, there exists a monic polynomial $h(X)=\sum a_{i} X^{i} \in$ $K[X]$ such that $0=h(\rho(Y))=\sum a_{i} \rho(Y)^{i}=\rho\left(\sum a_{i} Y^{i}\right)$. Being an embedding, $\rho$ is necessarily one-to-one, whence $\sum a_{i} Y^{i}=0$, contradicting the transcendence of $Y$, thus completing the proof that $(1) \Rightarrow(2)$.

(b) For (ii) $\Rightarrow($ i), argue that at least one suitable $\rho$ exists as in the proof of $(2) \Rightarrow(1)$. To establish the uniqueness of $\rho$, note (by the "purely inseparable" hypothesis) that 
each element $\alpha \in L$ has a minimum polynomial over $K$, say $h(X)$, with a unique root in $F$ and $\rho$ must send $\alpha$ to this root. (Indeed, if $h(X)=\sum a_{i} X^{i} \in K[X]$, then $h(\rho(\alpha))=\sum a_{i} \rho(\alpha)^{i}=\rho\left(\sum a_{i} \alpha^{i}\right)=\rho(0)=0$. $)$

(i) $\Rightarrow$ (ii). Assume (i). In particular, (1) holds. As we have shown that (1) $\Rightarrow(2)$, $L$ is algebraic over $K$. Take $F$ to be an algebraic closure of $K$, with $\tau: K \rightarrow F$ as the inclusion map. If the assertion fails, some element $\alpha \in L$ has a minimum polynomial over $K$, say $h(X)$, with at least two distinct roots, say $\alpha \neq \beta$, in $F$. Then a standard fact about splitting fields (cf. [24, Theorem 2.8, page 233]) yields embeddings $\rho_{1}, \rho_{2}: L \rightarrow F$ that extend $\tau$ and satisfy $\rho_{1}(\alpha)=\alpha$ and $\rho_{2}(\alpha)=\beta$. As $\alpha \neq \beta$, we have $\rho_{1} \neq \rho_{2}$, contradicting the uniqueness in (i), to complete the proof.

The next result removes the restrictions to nonfields that appeared in Theorem 2.1 (and Theorem 2.2). Notice, in particular, that the hypotheses of Theorem 2.5 allow the possibility that the PVD, $R$, is a field. For background on radicial homomorphisms, see [20, pages 246-249].

Theorem 2.5. Let $(R, M)$ be a PVD with associated valuation domain $V=\mathscr{T}(R)=(M: M)$ and, as usual, let $i_{R}: R \rightarrow V$ denote the inclusion map. Then

(a) the following seven conditions are equivalent:

(1) for each sl-homomorphism $f: R \rightarrow(H, P)$ such that $H$ is a $\mathcal{T}$-domain and $H / P$ is an algebraically closed field, there exists an sl-homomorphism $g: V \rightarrow H$ such that $g i_{R}=f$;

(2) for each sl-homomorphism $f: R \rightarrow(H, P)$ such that $H$ is a valuation domain and $H / P$ is an algebraically closed field, there exists an sl-homomorphism $g$ : $V \rightarrow H$ such that $g i_{R}=f$

(3) for each sl-homomorphism $f: R \rightarrow H$ such that $H$ is an algebraically closed field, there exists an sl-homomorphism $g: V \rightarrow H$ such that $g i_{R}=f$;

(4) $R / M \hookrightarrow V / M$ is an algebraic field extension;

(5) $V$ is integral over $R$;

(6) $V$ is the integral closure of $R$ (in the quotient field of $R$ );

(7) each overring of $R$ is a $P V D$;

(b) the following five conditions are equivalent:

(i) for each sl-homomorphism $f: R \rightarrow(H, P)$ such that $H$ is a $\mathscr{T}$-domain and $H / P$ is an algebraically closed field, there exists a unique sl-homomorphism $g: V \rightarrow H$ such that $g i_{R}=f$;

(ii) for each sl-homomorphism $f: R \rightarrow(H, P)$ such that $H$ is a valuation domain and $H / P$ is an algebraically closed field, there exists a unique sl-homomorphism $g: V \rightarrow H$ such that $g i_{R}=f$;

(iii) for each sl-homomorphism $f: R \rightarrow H$ such that $H$ is an algebraically closed field, there exists a unique sl-homomorphism $g: V \rightarrow H$ such that $g i_{R}=f$;

(iv) $R / M \hookrightarrow V / M$ is a purely inseparable (algebraic) field extension;

(v) $V$ is radicial over $R$ (i.e., $i_{R}$ is a radicial homomorphism). 
Proof. (a) (7) $\Leftrightarrow(6)$. See [22, Proposition 2.7], [16, Proposition 4.2].

$(6) \Leftrightarrow(5)$. This is valid, more generally, for any valuation overring $V$ of a domain $R$.

$(5) \Leftrightarrow(4)$. This can be shown by a direct calculation or, alternatively, by applying a basic result about pullbacks (see [18, Corollary 1.5(5)], [12, Lemme 2]) to the pullback description of $R$ as $V \times_{V / M}(R / M)$.

$(4) \Rightarrow(1)$. Assume (4), and let $f: R \rightarrow(H, P)$ be a strong local homomorphism such that $H$ is a $\mathscr{T}$-domain and $H / P$ is an algebraically closed field. We must find an slhomomorphism $g: V \rightarrow H$ such that $g i_{R}=f$. If $H$ is not a field, the assertion follows (and, in fact, gives a unique such $g$ ) from [3, Theorem 2.4] (which applies since $\mathscr{T}(H)=$ $H$ by hypothesis). Thus, without loss of generality, we may assume that $H$ is an algebraically closed field. The "strong local" condition ensures that $f(M)=P=0$, and so by the universal mapping property of factor rings (cf. [24, page 89]), $f$ factors through $R / M$. In other words, if $\pi_{1}$ denotes the canonical projection $R \rightarrow R / M$, then there is an embedding $j: R / M \rightarrow H$ such that $j \pi_{1}=f$. For convenience of notation, we view $j$ as an inclusion map. Let $k: R / M \rightarrow V / M$ denote the canonical embedding; for convenience of notation, we view $k$ as an inclusion map as well. Since $V / M$ is algebraic over $R / M$ by (4) and $H$ is algebraically closed, an application of Lemma 2.4(a) produces an embedding $\rho$ such that $\rho k=j$. Furthermore, it is clear that if $\pi_{2}$ denotes the canonical projection $V \rightarrow V / M$, then $\pi_{2} i_{R}=k \pi_{1}$. Therefore, the ring homomorphism $g:=\rho \pi_{2}$ satisfies $g i_{R}=\rho \pi_{2} i_{R}=\rho k \pi_{1}=j \pi_{1}=f$. Moreover, $g$ is an sl-homomorphism, since $g(M)=\rho \pi_{2}(M)=\rho(0)=0=P$, as desired.

$(1) \Rightarrow(2) \Rightarrow(3)$. Trivial.

(3) $\Rightarrow(4)$. As above, view $V / M$ as an extension field of $R / M$ via the canonical embed$\operatorname{ding} k: R / M \hookrightarrow V / M$. Now, suppose the assertion fails. Choose $X$ to be an element of some transcendence basis of $V / M$ over $R / M$. Choose an algebraic closure $F$ of $R / M$; let $\tau: R / M \hookrightarrow F$ be an embedding, viewed for convenience as an inclusion map. As above, let $\pi_{1}: R \rightarrow R / M$ and $\pi_{2}: V \rightarrow V / M$ denote the canonical projection maps. Observe that the ring homomorphism $f:=\tau \pi_{1}$ from $(R, M)$ to $(F, 0)$ is a strong local homomorphism, since $f(M)=\tau \pi_{1}(M)=\tau(0)=0$. Therefore, by (3), there exists an sl-homomorphism $g: V \rightarrow F$ such that $g i_{R}=f$. As $g$ is an sl-homomorphism, $g(M)=0$, and so $g$ factors through $V / M$. Thus, there exists an embedding $\rho: V / M \rightarrow F$ such that $\rho \pi_{2}=g$. Since $Y:=\rho(X) \in F$, it must be that $Y$ is algebraic over $R / M$. Therefore, we can write $Y^{n}+$ $\sum_{i=0}^{n-1} \alpha_{i} Y^{i}=0$ for some finite sequence of elements $\alpha_{i} \in R / M$. Then, since $\rho$ is a ring homomorphism which fixes each element of $R / M$, we have

$$
0=\rho(X)^{n}+\sum_{i=0}^{n-1} \alpha_{i} \rho(X)^{i}=\rho\left(X^{n}+\sum_{i=0}^{n-1} \alpha_{i} X^{i}\right) .
$$

Since $\rho$ is an injection, it now follows that $0=X^{n}+\sum_{i=0}^{n-1} \alpha_{i} X^{i}$. This contradicts the transcendence of $X$ over $R / M$ and completes the proof of (a).

(b) (v) $\Leftrightarrow$ (iv). $V$ is radicial over $R$ if and only if the following two conditions hold: the canonical map $\operatorname{Spec}(V) \rightarrow \operatorname{Spec}(R)$ is a bijection; and if $Q \in \operatorname{Spec}(V)$ and $P:=Q \cap R$, then the canonical field extension $\mathrm{qf}(R / P) \hookrightarrow \mathrm{qf}(V / Q)$ is purely inseparable (algebraic) 
(cf. [20, Proposition 3.7.1, page 246]). Now, the first of these conditions holds since $\operatorname{Spec}(R)=\operatorname{Spec}(V)$. Moreover, if $Q$ is a nonmaximal prime ideal of $V$ and $P:=Q \cap R$, then $q f(R / P)=\mathrm{qf}(V / Q)$ since $R_{P}=V_{Q}[21$, Proposition 2.6]. Thus, the second condition is, in the present situation, equivalent to (iv).

(iv) $\Rightarrow$ (i). Modify the above proof of $(4) \Rightarrow(1)$ in part (a) as follows. The earlier proof establishes the existence of at least one sl-homomorphism $g$ with the desired properties. To prove uniqueness, suppose that $H$ is an algebraically closed field (without loss of generality) and $h$ is an(other) sl-homomorphism $h: V \rightarrow H$ such that $h i_{R}=f$. Let $\pi_{1}, \pi_{2}$, and $k$ be as in the earlier proof. As in that proof, the "strong local" nature of $f$ ensures the existence of a unique ring homomorphism $j: R / M \rightarrow H$ such that $j \pi_{1}=f$. (For convenience of notation, view $k$ and $j$ as inclusion maps.) Similarly, since $h(M)=P=0$, the "strong local" nature of $h$ ensures the existence of a unique ring homomorphism $\rho: V / M \rightarrow H$ such that $\rho \pi_{2}=h$. Now, if $r \in R$, then

$$
\begin{aligned}
\rho k(r+M) & =\rho k \pi_{1}(r)=\rho \pi_{2} i_{R}(r)=\rho \pi_{2}(r) \\
& =h(r)=h i_{R}(r)=f(r)=j \pi_{1}(r)=j(r+M) .
\end{aligned}
$$

Hence, $\rho k=j$. As we are assuming in (iv) that $V / M$ is purely inseparable over $R / M$, it follows from Lemma 2.4(b) that the embedding $\rho$ is uniquely determined. Therefore, so is $\rho \pi_{2}=h$, as desired.

(i) $\Rightarrow$ (ii) $\Rightarrow$ (iii). Trivial.

(iii) $\Rightarrow$ (iv). Assume (iii). Then (3) also holds, and so by part (a), $V / M$ is algebraic over $R / M$. Let $\pi_{1}, \pi_{2}$, and $k$ be as above. By Lemma 2.4(b), it suffices to prove that if $\tau: R / M \rightarrow$ $F$ is an embedding of $R / M$ in an algebraically closed field $F$ and $\rho_{1}, \rho_{2}$ are embeddings $V / M \rightarrow F$ such that $\rho_{1} k=\tau=\rho_{2} k$, then $\rho_{1}=\rho_{2}$. Consider the ring homomorphisms $f$ : $R \rightarrow F$ and $g_{1}, g_{2}: V \rightarrow F$ defined by $f:=\tau \pi_{1}$ and $g_{i}:=\rho_{i} \pi_{2}$ (for $i=1,2$ ). Notice that $f, g_{1}$ and $g_{2}$ are each strong local homomorphisms. Moreover, $g_{1} i_{R}=\rho_{1} \pi_{2} i_{R}=\rho_{1} k \pi_{1}=\tau \pi_{1}=$ $f$; similarly, $g_{2} i_{R}=f$. Consequently, by (iii), $g_{1}=g_{2}$. In other words, $\rho_{1} \pi_{2}=\rho_{2} \pi_{2}$. As $\pi_{2}$ is surjective, it follows that $\rho_{1}=\rho_{2}$, to complete the proof.

In our last main result, Theorem 2.8, we will alter the context of Theorems 2.1,2.2, and 2.5 by weakening the sl-homomorphism condition. Mindful of Example 2.3, we should not focus on local homomorphisms, but rather on the following new concept.

Definition 2.6. If $(R, M)$ and $(S, N)$ are each quasilocal domains, then a ring homomorphism $f: R \rightarrow S$ is called a radical local homomorphism (rl-homomorphism) if $f(M) \subseteq N$ and for each $x \in N$, there exists a positive integer $t$ such that $x^{t} \in f(M)$.

The above definition of rl-homomorphisms was designed to be useful in studying almost pseudovaluation domains. Recall from [11] that a quasilocal domain $(R, M)$ is called an almost pseudovaluation domain (for short, APVD) if $(V, N):=\left(M:_{K} M\right)$ is a valuation domain in which $M$ is an $N$-primary ideal; that is, in which $\operatorname{Rad}_{T}(M)=N$. (As usual, we 
will let $\operatorname{Rad}_{A}(I)$ denote the radical of an ideal $I$ in a ring $A$.) Each PVD is an APVD. For examples of APVDs that are not PVDs in each Krull dimension $\geq 2$, see [13, Example 3.7(1)].

Remarks 2.7. (a) sl-homomorphism $\Rightarrow r l$-homomorphism $\Rightarrow$ local homomorphism; none of these implications is reversible.

(b) The composite of any rl-homomorphisms is an rl-homomorphism.

(c) If $R$ is an APVD, then the inclusion map $i_{R}: R \hookrightarrow \mathscr{T}(R)$ is an rl-homomorphism.

We next give an APVD-theoretic analogue of Theorem 2.5.

Theorem 2.8. Let $(R, M)$ be a APVD, with $(V, N):=(M: M)=: \mathscr{T}(R)$ and, as usual, $i_{R}$ : $R \rightarrow V$ denoting the inclusion map. Then

(a) the following six conditions are equivalent:

(1) for each rl-homomorphism $f: R \rightarrow(H, P)$ such that $H$ is a root-closed $\mathscr{T}$-domain and $H / P$ is an algebraically closed field, there exists an rl-homomorphism $g$ : $V \rightarrow H$ such that $g i_{R}=f$

(2) for each rl-homomorphism $f: R \rightarrow(H, P)$ such that $H$ is a valuation domain and $H / P$ is an algebraically closed field, there exists an rl-homomorphism $g$ : $V \rightarrow H$ such that $g i_{R}=f$

(3) for each rl-homomorphism $f: R \rightarrow H$ such that $H$ is an algebraically closed field, there exists an rl-homomorphism $g: V \rightarrow H$ such that $g i_{R}=f$;

(4) $R / M \hookrightarrow V / N$ is an algebraic field extension;

(5) $V$ is integral over $R$;

(6) $V$ is the integral closure of $R$ (in the quotient field of $R$ );

(b) the following four conditions are equivalent:

(i) for each rl-homomorphism $f: R \rightarrow(H, P)$ such that $H$ is a root-closed $\mathscr{T}$ domain and $H / P$ is an algebraically closed field, there exists a unique rlhomomorphism $g: V \rightarrow H$ such that $g i_{R}=f$;

(ii) for each rl-homomorphism $f: R \rightarrow(H, P)$ such that $H$ is a valuation domain and $H / P$ is an algebraically closed field, there exists a unique rl-homomorphism $g: V \rightarrow H$ such that $g i_{R}=f$;

(iii) for each rl-homomorphism $f: R \rightarrow H$ such that $H$ is an algebraically closed field, there exists a unique rl-homomorphism $g: V \rightarrow H$ such that $g i_{R}=f$;

(iv) $R / M \hookrightarrow V / N$ is a purely inseparable (algebraic) field extension;

(c) assume, in addition, that $R$ has Krull dimension at most 1 . Then the four equivalent conditions in (b) are equivalent to each of the following two conditions:

(v) $V$ is radicial over $R$ (i.e., $i_{R}$ is a radicial homomorphism) and $V$ is integral over $R$;

(vi) $V$ is radicial over $R$ (i.e., $i_{R}$ is a radicial homomorphism) and $V$ is the integral closure of $R$ (in the quotient field of $R$ ).

Proof. (a) We noted in the proof of Theorem 2.5 (a) that $(6) \Leftrightarrow(5)$ on general principles. Moreover, it is clear that $(5) \Rightarrow(4)$. We show next that $(4) \Rightarrow(5)$. Assume (4), and consider 
Ahmed Ayache et al. 9

any element $v \in V$. As $v+N \in V / N$ is algebraic over $R / M$, we have

$$
\xi:=v^{n}+r_{n-1} v^{n-1}+\cdots+r_{1} v+r_{0} \in N
$$

for some finite sequence of elements $r_{i} \in R$. However, $M$ is $N$-primary (since $R$ is an APVD), and so some integral power of $\xi$ belongs to $M$. Expanding such a power leads to an integrality equation for $v$ over $R$, and so (5) holds.

$(4) \Rightarrow(1)$. We adapt the proof of the corresponding implication in Theorem 2.5(a). Assume (4). Suppose that $(H, P)$ and $f$ are as in (1). In proving the existence of a suitable rl-homomorphism $g$, we treat two cases.

In the first case, there exists $m \in M$ such that $f(m) \neq 0$. At this point, we adapt the construction in the proof of [3, Theorem 2.4]. For each $z \in V$, let $g(z):=f(z m) / f(m)$. We claim that $g(z) \in H$. Since $H$ is a $\mathscr{T}$-domain, it suffices to prove that $g(z) \in(P: P)$; equivalently, that $y:=g(z) w \in P$ for each $w \in P$. By the "radical local" property of $f$, there exists a positive integer $n$ such that $w^{n} \in f(M)$. As $z M \subseteq M$, it follows that

$$
y^{n} \in \frac{1}{f\left(m^{n}\right)} f\left(m^{n} z^{n} M\right)=\frac{1}{f\left(m^{n}\right)} f\left(m^{n}\right) f\left(z^{n} M\right)=f\left(z^{n} M\right) \subseteq f(M) \subseteq P \subset H .
$$

Since $H$ is assumed to be a root-closed domain, we conclude that $y \in H$. As $y^{n} \in P$, we have $y \in \operatorname{Rad}_{H}(P)=P$, thus proving the above claim.

We now have a function $g: V \rightarrow H$. As in the proof of [3, Theorem 2.4], one checks that $g$ is a ring homomorphism such that $g i_{R}=f$. For this case, it remains only to establish the "radical local" property for $g$. If $v \in N$, then since $R$ is an APVD, there exists a positive integer $t$ such that $v^{t} \in M$, whence $g(v)^{t}=g\left(v^{t}\right) \in g(M)=f(M) \subseteq P$ and $g(v) \in \operatorname{Rad}_{H}(P)=P$. Moreover, if $p \in P$, the fact that $f$ is a radical local homomorphism provides a positive integer $s$ and an element $m^{*} \in M$ such that $p^{s}=f\left(m^{*}\right)$. Since $g i_{R}=f$ and $M \subseteq N$, it follows that $p^{s}=g\left(m^{*}\right)$, and so $g$ is radical local, to complete the proof of the first case.

In the second case, $f(M)=0$. As $f$ is an rl-homomorphism, every element of $P$ is nilpotent. Thus, since $H$ is a domain, $P=0$, and so $H$ is an algebraically closed field. Then, as in the proof of Theorem 2.5(a), we can use Lemma 2.4(a) to find an sl-homomorphism $g: V \rightarrow H$ such that $g i_{R}=f$. By Remarks 2.7(a) (or since $g$ is unital and $H$ is a field), $g$ is an rl-homomorphism, as desired.

$(1) \Rightarrow(2) \Rightarrow(3)$. Trivial.

$(3) \Rightarrow(4)$. The following comments explain how to modify the proof of the corresponding implication in Theorem 2.5(a). Replace considerations of $V / M$ throughout with $V / N$. As $f:=\tau \pi_{1}$ is a strong local homomorphism, it is also a radical local homomorphism. After obtaining a radical local homomorphism $g: V \rightarrow F$ such that $g i_{R}=f$, infer from its "radical local" property that $g(N)=0$, whence $g$ factors through $V / N$ via an embedding $\rho: V / N \rightarrow F$ such that $\rho \pi_{2}=g$. The remainder of the proof proceeds as before, mutatis mutandis.

(b) We sketch how to adapt the above proofs. 
(iv) $\Rightarrow$ (i). Modify the above proof of $(4) \Rightarrow(1)$ in part (a) as follows. The earlier proof establishes the existence of at least one rl-homomorphism $g$ with the desired properties. If $f(M) \neq 0$, there is at most one such $g$, as it must satisfy the earlier formula $g(z)=$ $f(z m) / f(m)$ (for any nonzero $m \in M$, since $g$ is required to extend $f$ ). Thus, in proving uniqueness, we may suppose, without loss of generality, that $H$ is an algebraically closed field, and we consider an(other) rl-homomorphism $h: V \rightarrow H$ such that $h i_{R}=f$. For the rest of the argument, see the proof of (iv) $\Rightarrow$ (i) in Theorem 2.5(b), replacing occurrences of "strong local" with "radical local" and " $V / M$ " with " $V / N$."

(i) $\Rightarrow$ (ii) $\Rightarrow$ (iii). Trivial.

(iii) $\Rightarrow$ (iv). It suffices to modify the proof of (iii) $\Rightarrow$ (iv) in Theorem 2.5(b) as follows: replace occurrences of " $V / M$ " with " $V / N$, and observe that $f, g_{1}$ and $g_{2}$ are each radical local homomorphisms.

(c) $(\mathrm{vi}) \Leftrightarrow(\mathrm{v})$. See the above comments concerning $(6) \Leftrightarrow(5)$.

(v) $\Rightarrow$ (iv). Since $N \cap R=M$, it suffices to apply the standard characterization of "radicial" that was mentioned in the proof of $(\mathrm{v}) \Leftrightarrow$ (iv) in Theorem 2.5(b).

(iv) $\Rightarrow(v)$. Assume (iv). Then, in particular, $V / N$ is algebraic over $R / M$. By the first paragraph of the proof of (a), $V$ is integral over $R$. Since $V$ is an overring of $R$ and the hypothesis on Krull dimension means that $M$ is the only (possibly) nonzero prime ideal of $R$, it follows that if $Q \in \operatorname{Spec}(V)$ and $P:=Q \cap R$, then $\mathrm{qf}(V / Q)$ is purely inseparable (algebraic) over qf $(R / P)$. Therefore, by the above-mentioned characterization of "radicial," it suffices to prove that the canonical function $\operatorname{Spec}(V) \rightarrow \operatorname{Spec}(R)$ is a bijection. This, in turn, is evident since the "incomparable" property of integrality ensures that $N$ is the only (possibly) nonzero prime ideal of $V$. The proof is complete.

Remarks 2.9. (a) In view of Theorem 2.5(a), it is reasonable to ask if the six equivalent conditions (1)-(6) in Theorem 2.8(a) are also equivalent to the following condition:

(7) each overring of (the APVD $R$ ) is an APVD.

In fact, the answer is in the negative, for Badawi and Houston [11, Example 3.9] have given an example of a one-dimensional APVD, $(R, M)$, whose integral closure is the valuation domain $V:=(M: M)$, although some (integral) overring of $R$ is not an APVD. We see from that example that, despite expectations possibly raised by Theorem $2.5(\mathrm{~b})$, the six equivalent conditions (i)-(vi) in Theorem 2.8(c) also fail to be equivalent to condition (7). However, it follows from [11, Propositions 3.8 and 3.10] that, under the hypotheses of Theorem 2.8, condition (7) is equivalent to the conjunction of any of the ten conditions (1)-(6), (i)-(iv) with the condition that each integral overring of $R$ is an APVD.

(b) The above work can be used to motivate a program of domain-theoretic studies with a categorical flavor. For instance, it is interesting that the integrality of $\mathscr{T}(R)$ over a PVD $R$, which Theorem 2.5(a) shows is equivalent to the categorical requirement that certain extensions of ring homomorphisms exist, is also equivalent to the requirement that each overring of $R$ be a PVD. (cf. also Remarks 2.9(a).) We believe that it would be valuable to seek categorical characterizations for many of the other instances where important classes of domains are known to be stable under the formation of overrings.

In addition, in the spirit of Theorem 2.8, it would be interesting to seek other analogues of Theorem 2.5 for some divided domains besides the APVDs. No doubt, this 
would involve introducing variants of the sl- and rl-homomorphism concepts and developing factorization results for them in the spirit of the work done for sl-homomorphisms in [3, second half of Section 3].

\section{References}

[1] D. F. Anderson, A. Badawi, and D. E. Dobbs, Pseudo-valuation rings. II, Bollettino della Unione Matematica Italiana. Serie VIII. Sezione B. Articoli di Ricerca Matematica 3 (2000), no. 2, 535545.

[2] D. F. Anderson and D. E. Dobbs, Pairs of rings with the same prime ideals, Canadian Journal of Mathematics 32 (1980), no. 2, 362-384.

[3] A. Ayache, D. E. Dobbs, and O. Echi, Reflection of some quasi-local domains, Journal of Algebra and Its Applications 5 (2006), no. 2, 201-213.

[4] A. Badawi, A visit to valuation and pseudo-valuation domains, Zero-Dimensional Commutative Rings (Knoxville, Tenn, 1994), Lecture Notes in Pure and Appl. Math., vol. 171, Dekker, New York, 1995, pp. 155-161.

[5] _ On $\Phi$-pseudo-valuation rings. II, Houston Journal of Mathematics 26 (2000), no. 3, 473-480.

[6] _ On chained overrings of pseudo-valuation rings, Communications in Algebra 28 (2000), no. 5, 2359-2366.

[7] _ On $\phi$-chained rings and $\phi$-pseudo-valuation rings, Houston Journal of Mathematics 27 (2001), no. 4, 725-736.

[8] _ On divided rings and $\phi$-pseudo-valuation rings, International Journal of Commutative Rings 1 (2002), no. 2, 51-60.

[9] _ Pseudo-valuation domains: a survey, Mathematics \& Mathematics Education (Bethlehem, 2000), World Scientific, New Jersey, 2002, pp. 38-59.

[10] A. Badawi, D. F. Anderson, and D. E. Dobbs, Pseudo-valuation rings, Commutative Ring Theory (Fès, 1995), Lecture Notes in Pure and Appl. Math., vol. 185, Dekker, New York, 1997, pp. 5767.

[11] A. Badawi and E. G. Houston, Powerful ideals, strongly primary ideals, almost pseudo-valuation domains, and conducive domains, Communications in Algebra 30 (2002), no. 4, 1591-1606.

[12] P.-J. Cahen, Couples d'anneaux partageant un idéal [Pairs of rings that share an ideal], Archiv der Mathematik (Basel) 51 (1988), no. 6, 505-514.

[13] G. W. Chang, H. Nam, and J. Park, Strongly primary ideals, Arithmetical Properties of Commutative Rings and Monoids, Lecture Notes Pure Appl. Math., vol. 241, Chapman \& Hall/CRC, Florida, 2005, pp. 378-388.

[14] Y. H. Cho, Pseudo-valuation domains, Korean Mathematical Society. Communications 11 (1996), no. 2, 281-284.

[15] _ Pseudo valuation rings, Honam Mathematical Journal 23 (2001), no. 1, 21-28.

[16] D. E. Dobbs, Coherence, ascent of going-down, and pseudo-valuation domains, Houston Journal of Mathematics 4 (1978), no. 4, 551-567.

[17] D. E. Dobbs, M. Fontana, J. A. Huckaba, and I. J. Papick, Strong ring extensions and pseudovaluation domains, Houston Journal of Mathematics 8 (1982), no. 2, 167-184.

[18] M. Fontana, Topologically defined classes of commutative rings, Annali di Matematica Pura ed Applicata. Serie Quarta 123 (1980), 331-355.

[19] H. Gebru, Krull's conjecture, pseudo-valuation domains and PF-dimension, Mathematics Journal of Toyama University 21 (1998), 153-161.

[20] A. Grothendieck and J. A. Dieudonné, Eléments de Géométrie Algébrique. I, Springer, New York, 1971. 
12 Universal mapping properties of pseudovaluation domains

[21] J. R. Hedstrom and E. G. Houston, Pseudo-valuation domains, Pacific Journal of Mathematics 75 (1978), no. 1, 137-147.

[22] __ Pseudo-valuation domains. II, Houston Journal of Mathematics 4 (1978), no. 2, 199207.

[23] B. G. Kang and M. H. Park, Completion of a globalized pseudo-valuation domain, Houston Journal of Mathematics 28 (2002), no. 4, 701-710.

[24] S. Lang, Algebra, 3rd ed., Addison-Wesley, Massachusetts, 1993.

[25] R. Matsuda and T. Sugatani, Cancellation ideals in pseudo-valuation domains, Communications in Algebra 23 (1995), no. 11, 3983-3991.

[26] A. Okabe, Some ideal-theoretical characterizations of divided domains, Houston Journal of Mathematics 12 (1986), no. 4, 563-577.

Ahmed Ayache: Department of Mathematics, Faculty of Sciences, University of Bahrain, P.O. Box 32038, Isa Town, Bahrain

E-mail address: aayache@sci.uob.bh

David E. Dobbs: Department of Mathematics, University of Tennessee, Knoxville,

TN 37996-1300, USA

E-mail address: dobbs@math.utk.edu

Othman Echi: Department of Mathematics, Faculty of Sciences of Tunis, University Tunis-El Manar, Campus Universitaire, 2092 Tunis, Tunisia

E-mail addresses: othechi@yahoo.com; othechi@math.com 


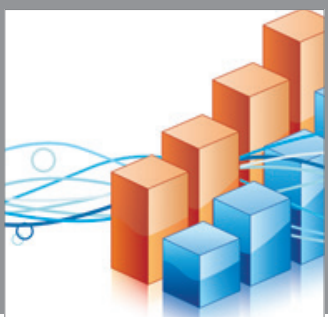

Advances in

Operations Research

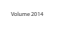

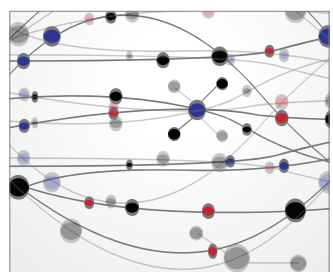

\section{The Scientific} World Journal
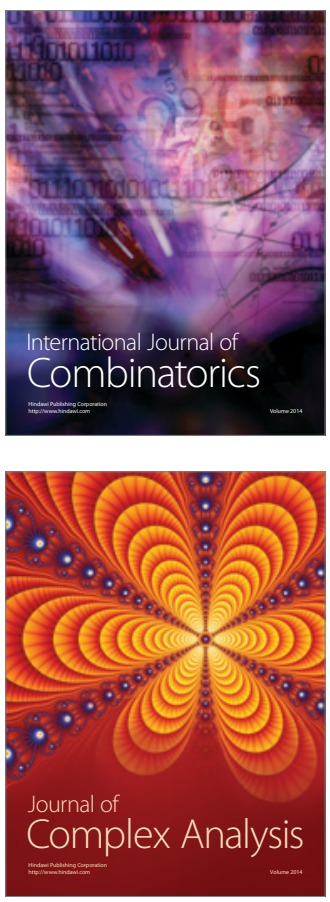

International Journal of

Mathematics and

Mathematical

Sciences
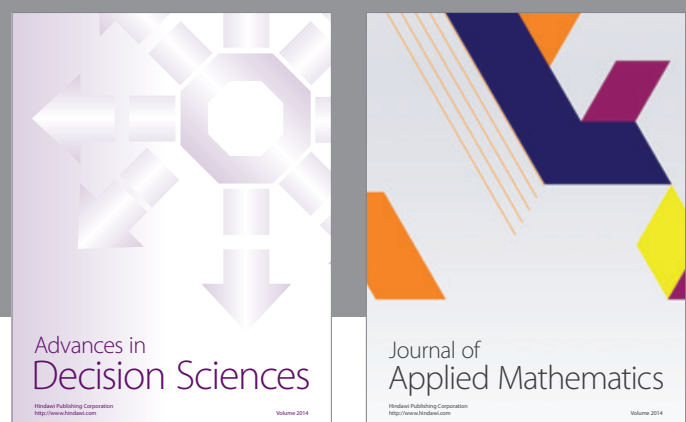

Journal of

Applied Mathematics
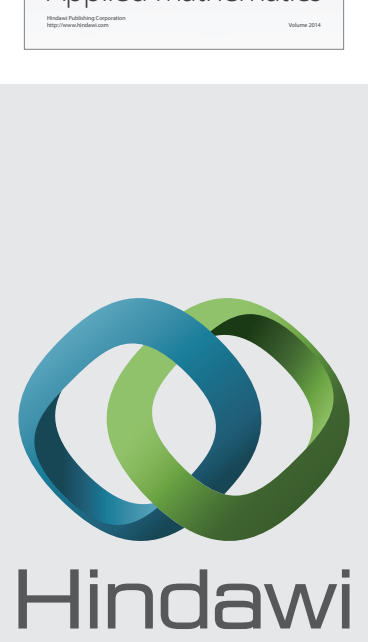

Submit your manuscripts at http://www.hindawi.com
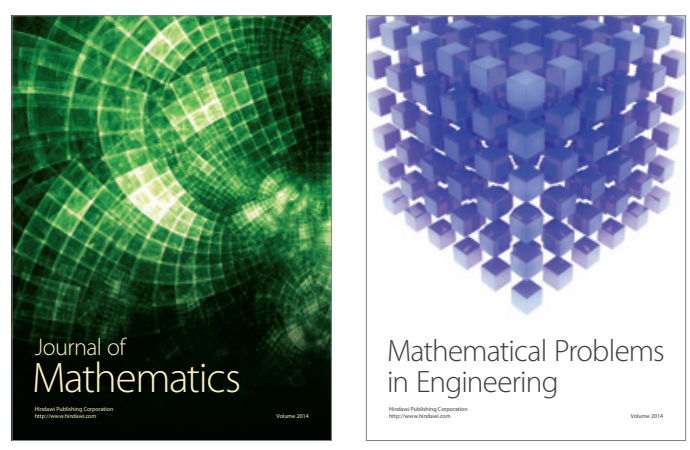

Mathematical Problems in Engineering
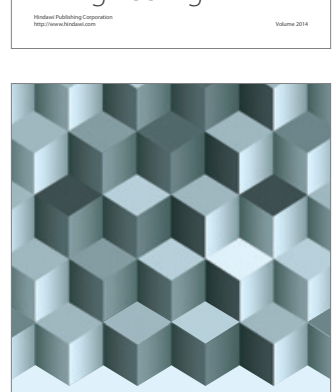

Journal of

Function Spaces
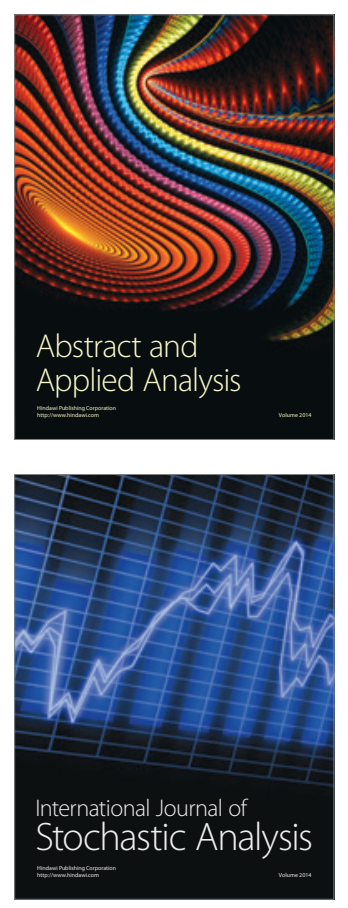

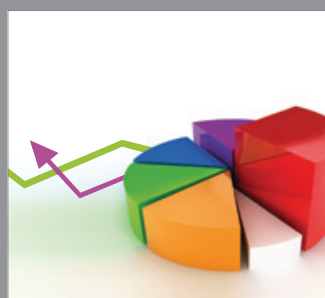

ournal of

Probability and Statistics

Promensencen
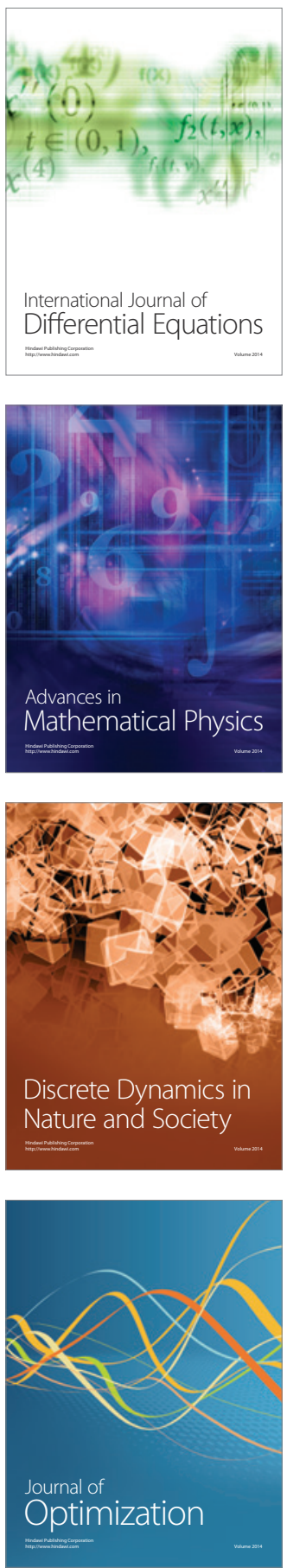